\title{
On the role of sea-ice transport in modifying Arctic responses to global climate change
}

\author{
James Maslanik, Jeremy Dunn \\ Colorado Center for Astrodynamics Research, University of Colorado, Boulder, CO 80309, U.S.A.
}

\begin{abstract}
The role of dynamics in modifying the response of the Arctic ice pack to inter-annually varying forcings and to climate perturbations is investigated using simulations from a two-dimensional ice model and a global climate model (GCM). Inter-annual variability in ice-covered area for 1985-93 is dominated by ice transport, and different transport regimes affect substantially the response of the ice pack to climate perturbations. The thermodynamic-only simulations are more sensitive to initial ice conditions, and respond less than the dynamic-thermodynamic model to small perturbations, but with a greater response to larger perturbations. Comparisons of GCM simulations that use different ice treatments highlights the importance of considering the distribution of ice thickness and extent in assessing climate-change responses.
\end{abstract}

\section{INTRODUCTION}

Based on global climate model (GCM) simulations, the coverage of polar sea ice is expected to be quite sensitive to global warming. In these simulations, a main factor controlling ice response is the positive feedback between sea-ice cover and albedo. Accurate simulations of the expected climate response to warming thus depend on the realism with which climate models simulate the change in ice extent under conditions of enhanced melt. The inclusion of ice transport improves agreement with observations and is likely to affect the response of the ice cover to climate perturbations (Hibler and Ackley, 1983).

Here, we examine further the role of ice dynamics in modifying the changes in Arctic ice fraction and thickness under different climate scenarios, and consider how the inclusion of dynamics might modify the response of sea ice to warming.

\section{APPROACH}

To investigate the response of the Arctic ice to inter-annual variability under different climate conditions, a stand-alone two-dimensional (2-D) ice model is run in dynamicthermodynamic and thermodynamic-only modes using daily and inter-annually varying atmospheric forcings. Control simulations using forcings for 1985-93 are compared to results using perturbed longwave flux and surface air temperature. Results are then applied to the interpretation of responses of the Arctic ice cover in GCM simulations.

Specific questions are: (a) how much of the inter-annual variability in the sea-ice cover is attributable to variations in thermodynamic forcings vs ice transport? (b) does the response of the ice cover to air temperature and radiation perturbations vary as a function of large-scale ice-transport patterns? (c) does the inclusion of ice dynamics change significantly the response of the ice cover to perturbations in radiation and air temperature typical of climatic-change scenarios? and (d) how sensitive is the thermodynamic-only vs dynamic-thermodynamic ice response to baseline icecover conditions?

\section{Models and forcing fields}

The 2-D ice model used here is a derivative of the basic 2-D dynamic-thermodynamic model with a viscous-plastic ice rheology developed by Hibler (1979), and is similar to versions used in a variety of studies of sea-ice processes and sensitivities (e.g. Walsh and Zwally, 1990; Holland and others, 1993). Modifications include separate thermodynamic calculations for first-year ice, second-year ice, and multi-year ice (Walsh and Zwally, 1990); an approximation of a thickness distribution for growth-rate calculations (Walsh and others, 1985); and stability and fetch-adjusted turbulent fluxes (Maslanik and Key, 1995; Ebert and Curry, 1993). Albedo is estimated as a function of snow and ice thickness and melt-pond conditions (Ebert and Curry, 1993), with melt-pond albedo based on prescribed melt-pond depth and area that vary with ice type and duration of melt. A slab mixed-layer ocean is included that acts as a heat reservoir in areas with no ice cover. Radiative fluxes, surface air temperatures, and winds are provided as external forcings as described below. Ocean flux and average annual geostrophic currents from an ice-ocean model (Hibler and Bryan, 1987) are used as in Flato and Hibler (1992). Precipitation is prescribed from climatology. The model domain includes the Arctic Ocean and adjacent seas (Fig. 1). Cell spacing of the Cartesian grid is $80 \mathrm{~km}$. Eighteen year simulations were performed by cycling twice through the nine years of forcing data using a 12 hours time-step.

The forcing fields were obtained from the National Center for Environmental Prediction (NCEP) National Center for Atmospheric Research (NCAR) Reanalysis Project for 1985-93 (Kalnay and others, 1996) and include downwelling longwave $\left(F_{\mathrm{L}}\right)$ and shortwave radiation 

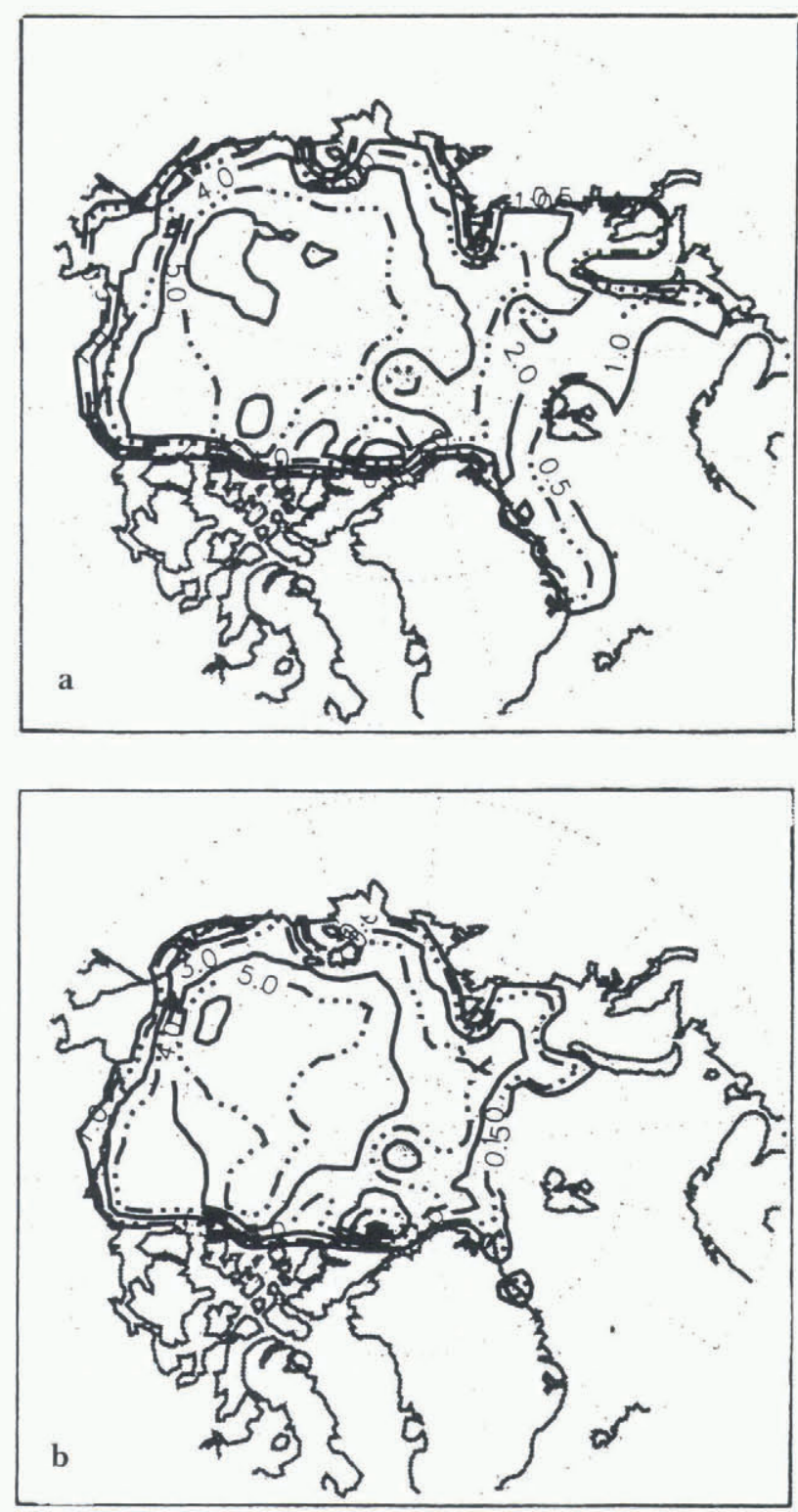

Fig. 1. Nine year mean ice thickness for March (a) and September (b) as simulated using a TO ice model and NCEP forcings.

$\left(F_{\mathrm{R}}\right)$, surface air temperatures $\left(T_{\mathrm{A}}\right)$, and sea-level pressures (SLP). Overall, the NCEP fields appear quite reasonable in terms of basic patterns and seasonal cycles. Monthly means averaged over the ice-model domain generally compare well with climatologies. However, $F_{\mathrm{R}}$ is greater than observations suggest, with a June mean at the North Pole of $362 \mathrm{Wm}^{-2}$. Also, $T_{\mathrm{A}}$ is greater than in the comparison data, mainly in spring. Overall, though, the NCEP data provide the best available suite of internally consistent and interannually varying forcings for the types of model experiments discussed here.

The NCEP data were mapped to the model grid using a Gressman distance-weighted interpolation. Geostrophic winds were calculated from the NCEP SLP. With these NCEP forcings, the ice model consistently produced an ice cover with realistic ice-advection patterns, but with an overestimate of summer melt when representative ice albedos were used. $F_{\mathrm{R}}$ was then reduced by a factor of 0.1 for a better match of the NCEP $F_{\mathrm{R}}$ with observations, and the NCEP temperatures for 15 May-30 June were replaced by climatological air temperatures (Crutcher and Meserve, 1970) to address the apparent positive bias in the NCEP air temper- atures in spring. These adjustments are consistent with the effects of an underestimate in Arctic cloud fraction in late spring and summer, which is seen in the NCEP data when compared to observations. The adjustments to $F_{\mathrm{R}}$ and $T_{\mathrm{A}}$ (and the perturbation of these forcings) introduce some imbalance relative to each other and to the unchanged SLP fields, but this inconsistency is assumed to be insignificant for the interpretations used here. Using these revised forcings, the ice cover is simulated well throughout the year in the dynamic-thermodynamic (DT) control run as discussed below, but with some remaining problems in the initial thermodynamic-only (TO) control run.

The stand-alone ice model ignores some other important features of the Arctic, including the effects of ocean dynamics on sea-ice anomalies and, in turn, many of the effects of ice transport and formation in modifying ocean salinity and temperature. An explicit ice-thickness distribution that includes ridging would also have an effect on the response of the ice pack to different climate scenarios (Flato and Hibler, 1995), as is noted below in comparing the effects of the TO and DT thickness distributions. Details of regional atmosphere-ocean couplings, such as intensification of low-pressure systems and modifications of storm tracks, are also neglected. We expect, though, that the simulations here capture the basic patterns of sea-ice responses that could be expected from GCMs using this same basic type of TO or DT ice treatment.

\section{Experiment design}

The experimental design consists of a set of 2-D simulations to examine how ice extent and volume, simulated with and without ice transport, respond to $F_{\mathrm{L}}$ and $T_{\mathrm{A}}$ perturbations under the different atmospheric conditions represented by the daily varying forcings for 1985-93. The second part of the experiment considers how the relationships between ice transport and atmospheric forcings affect the interpretation of GCM climate-change simulations.

The suite of perturbed climate simulations using the 2-D ice model include: (Scenario 1) $F_{\mathrm{L}} \times 1.03$; (Scenario 2) $T_{\mathrm{A}}+2.0^{\circ}$; (Scenario 3$) F_{\mathrm{L}} \times 1.03$ and $T_{\mathrm{A}}+2.0^{\circ}$; and (Scenario 4) $F_{\mathrm{L}} \times 1.05$ and $T_{\mathrm{A}}+2.0^{\circ}$. To separate the effects of inter-annually varying dynamic and thermodynamic forcings, an additional simulation (Scenario 5) was performed using the DT model with a nine year mean NCEP $F_{\mathrm{L}}$ and $T_{\mathrm{A}}$ "climatology" and inter-annually varying winds. Scenario 6 is the same as Scenario 1, but applied to the second of the two TO control runs, which is described below. The perturbations used are within the general range of mean changes predicted in doubled $\mathrm{CO}_{2}$ scenarios. However, since the perturbations are applied uniformly, the effects of regional differences are not addressed.

The GCM scenarios investigated are present-day $\mathrm{CO}_{2}$ and doubled $\mathrm{CO}_{2}$ with separate climate-model runs using TO and DT ice. These coupled atmosphere ice simulations were obtained using the NCAR GENESIS Global Environmental and Ecological Simulation of Interactive Systems) Earth system model (Pollard and Thompson, 1994). GENESIS version 1 is based on NCAR Community Climate Model (CCMl) and includes a cavitating fluid DT ice model (Flato and Hibler, 1992), a slab ocean, and a land-surface model. Version 2 of the global model performs relatively well for the Arctic (Maslanik and others, 1996a), and includes aspects of CCM2 with various modifications and im- 
provements to each Earth-system component. A principal difference between the model versions as used here is that the GENESIS version 1 simulations with dynamic sea ice were obtained using prescribed winds because of poorly simulated SLP fields, whereas the GENESIS version 2 results used interactively simulated winds. Model resolutions used are a spectral horizontal Atmospheric GCM (AGCM) grid of T31 (3.75 latitude and longitude) with a $2^{\circ} \times 2^{\circ}$ surface model. GENESIS version 1 results are available for $1 \times \mathrm{CO}_{2}$ and $2 \times \mathrm{CO}_{2}$ scenarios with DT and TO ice (Pollard and Thompson, 1994). Version 2 runs have been completed for the two $\mathrm{CO}_{2}$ scenarios with DT ice, and are in progress for the TO ice runs.

\section{RESULTS}

\section{Ice-model control runs}

Mean ice extent in March and September for the TO and DT ice-only model control runs (Figs 1 and 2) are similar (within $10 \%$ ) and reasonable, although overestimated in September. Magnitudes and inter-annual changes in ice
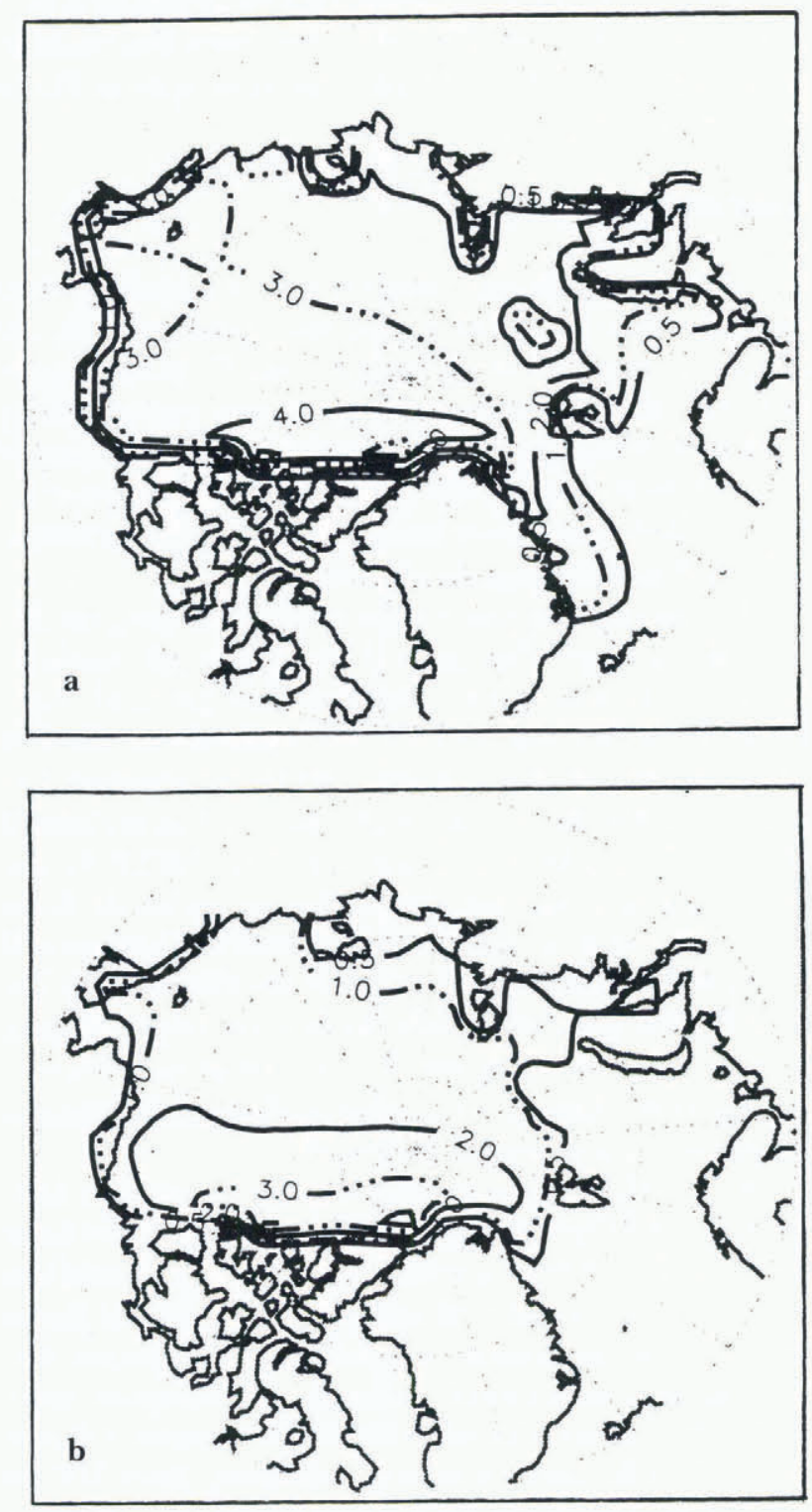

Fig. 2. Nine year mean ice thickness for March (a) and September (b) as simulated using a DT ice model and NCEP forcings.

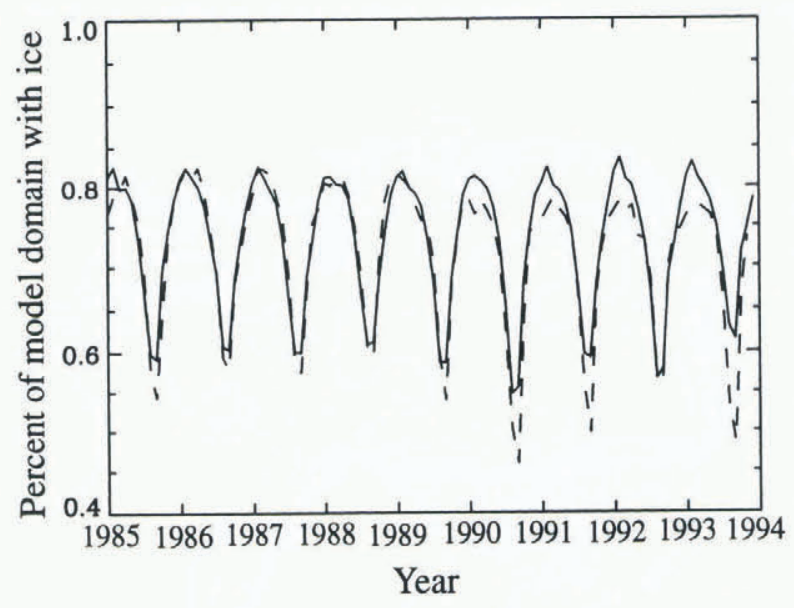

Fig. 3. Comparison of fraction of the Arctic Ocean and peripheral seas (the model domain in Fig. 1) covered by at least 15\% ice fraction as simulated in the DT control run (solid line) and as mapped using SMMR and SSM/I data (dashed line) (Maslanik and others, 1996b).

extent (area covered by at least $15 \%$ ice) generally follow the time series of SMMR and SSM/I derived ice coverage (Fig. 3). The DT ice thicknesses are realistic, but the TO ice is too thick in the central and western Arctic due to localized apparent biases in the NCEP air temperatures. As seen in the DT results, the inclusion of ice dynamics reduces the effects of such biases. Rather than further adjusting the forcings, a second TO control run ("Control 2") was performed with the TO model tuned by adjusting albedo and minimum open-water fraction to yield a thinner initial ice cover. As shown later, the responses to perturbations depend on initial ice thickness. This second control run was therefore included to represent the TO model response given more realistic initial ice thicknesses.

Inter-annual variability and climate-change response as a function of ice treatment in the ice-only model

Inter-annual variability in fractional cover of ice in the Arctic Ocean is considerably greater in the DT runs (Fig. 4) than in the TO simulations, varying by a maximum in

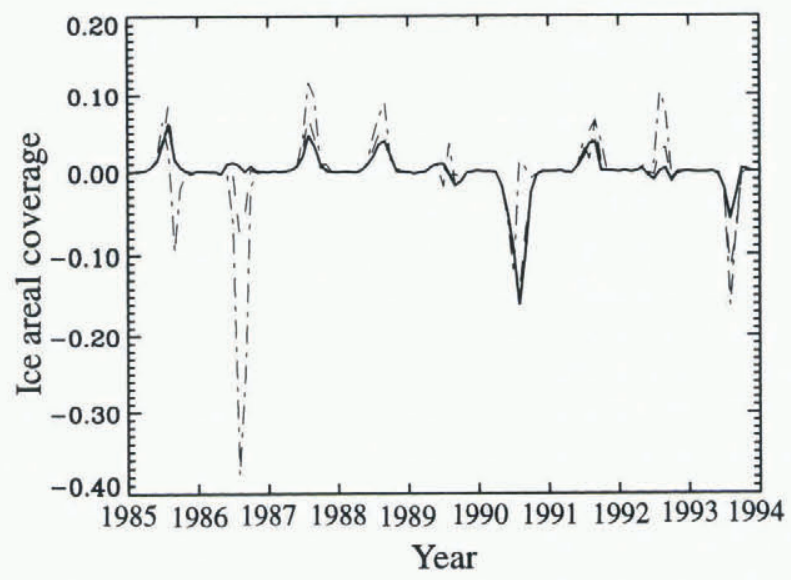

Fig. 4. Inter-annual variability in anomalies of sea-ice fractional coverage for the Arctic Ocean using the DT model. (control run is the solid line; climate scenario 1 is the dashed line; climate scenario 3 is the dash-dot line). Anomalies are the deviation of each individual monthly mean from the nine year mean for the control run and scenarios 1 and 3 . 
1993 of $16 \%$ of the mean summer ice fraction for the DT control run. Ice fractional coverage changes by $<1 \%$ in the TO Control 1 run and $4 \%$ in the TO Control 2 run for the nine year period. Ice volume (not shown) varies within $\pm 5 \%$ of the mean in the DT control run, except in 1993 when the summer anomaly is $-8 \%$. For the TO Control 1 volume, the maximum anomaly 1993 of $-3 \%$ occurs in December 1993 , with a variability of about $1 \%$ of the mean in other years, with similar variability in the Control 2 run. The DT simulation using inter-annually varying winds, but with the NCEP nine year climatology, yields a nearly identical variability, indicating that the inter-annual changes in ice cover are due mainly to ice-advection patterns rather than to short-term changes in air temperatures and radiative flux.

Interestingly, the temporal variability and relative magnitudes of these changes in ice fraction and ice volume under different climate scenarios change in relation to patterns of large-scale ice transport. In the DT control run, minimum ice extent occurs in 1990, consistent with observations of ice extent (Serreze and others, 1995; Maslanik and others, 1996b). However, when $F_{\mathrm{L}}$ and $T_{\mathrm{A}}$ are increased, the minimum ice fraction occurs in 1986 rather than 1990 (Fig. 4), with a concurrent change in ice volume (Fig. 5). The explanation for this is that in 1986, winds favor a more southward ice margin by advecting ice into the Siberian, Chukchi, and Beaufort seas from the central Arctic. In 1990, reduced ice extent in summer is due primarily to northward advection of ice.

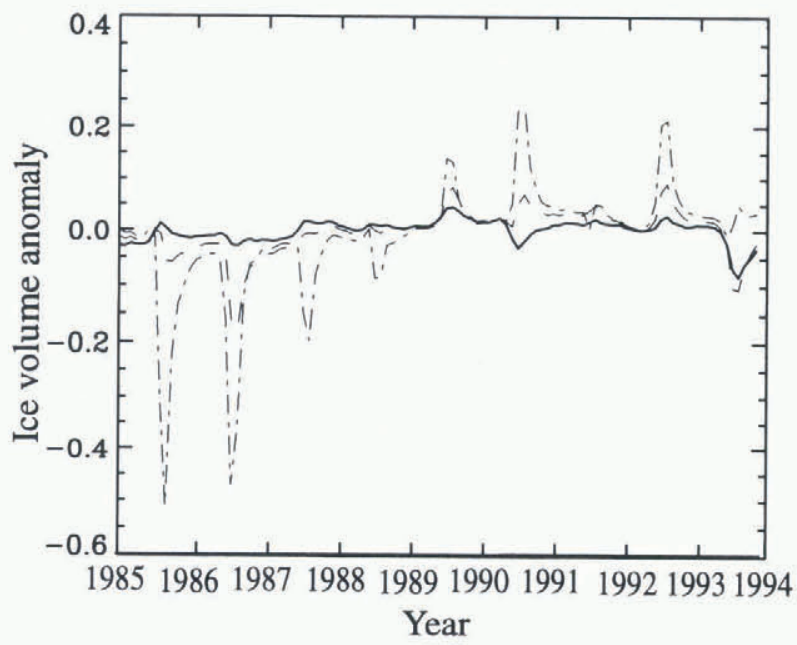

Fig. 5. Same as Figure 4, but for inter-annual variability in anomalies of sea-ice volume $\left(10^{4} \mathrm{~km}^{3}\right)$.

Ice advection thus affects the climate response of the pack in 1986, and to a lesser degree in 1985 , by placing ice in regions where melt is enhanced. In contrast, ice extent in 1990 is less affected by enhanced melt since advection already produces a more northward ice margin, and the ice has been moved to a region of less melt. For small perturbations, the southward drift typical of 1986 might mask the effects on ice extent of additional melt, but for larger perturbations in $T_{\mathrm{A}}$ and $F_{\mathrm{L}}$ the southward ice advection is overwhelmed by the added melt, with the net result being a thinner ice pack and reduced ice extent. In short, changes in ice extent and volume are affected by a close coupling between melt and transport, where certain atmospheric- circulation regimes might either enhance or reduce the response of the pack to changes in fluxes and air temperatures.

\section{Overall response to climate change using TO vs DT ice}

The decreases in nine year mean ice fractional coverage with increasing $F_{\mathrm{L}}$ and $T_{\mathrm{A}}$ are similar for the TO and DT runs, although the summer minimum TO ice extent is less sensitive than the DT ice extent to smaller perturbations. The thinner and slightly less extensive TO Control 2 ice pack (Scenario 6) is more sensitive than the thicker TO Control 1 ice. Ice fraction decreases by $22 \%$ and ice volume by $46 \%$. As perturbations become larger, such as in Scenario 3, the TO model responds more than the DT model, with a $10 \%$ greater decrease in ice fraction.

Differences between the TO and DT runs are greater in terms of ice volume. As with ice extent, the TO simulations are more responsive to large perturbations. For example, the decrease in DT volume is slightly greater than the TO decrease for the Scenario 1 perturbation, but the DT ice volume is relatively insensitive to the extra $F_{\mathrm{L}}$ perturbation in Scenario 4 compared to Scenario 3 - ice volume is only $13 \%$ less in the Scenario 4 run. For TO however, the difference between Scenarios 3 and 4 is $69 \%$. Scenario 4 removes all but $4 \%$ of the September ice volume in the TO simulation, while $21 \%$ of the control volume remains in the DT simulation. The results are similar for mean annual ice volume, with the exception that the TO ice volumes are more sensitive than the DT ice volume to all the perturbation scenarios.

These patterns of differences in sensitivity reflect the nature of the ice cover simulated using a TO vs a DT model. In a TO simulation, the Arctic sea ice tends toward a more uniform thickness distribution. In contrast, the ice cover simulated using a DT model will typically produce a bimodal thickness distribution that reflects ice thicknesses of 1-3 m over most of the basin, with much thicker ice confined to the Canadian sector of the Arctic in response to the general wind patterns. With the TO Control 1 ice cover, the thickness distribution implies that a larger perturbation is required to reduce the ice thickness enough to produce large changes in ice extent. The DT ice cover, with its greater proportion of thinner ice, can respond more easily to smaller perturbations. A climate model with a TO ice model that produces an overly thick ice cover in a control run will be less sensitive to small changes in radiative fluxes or air temperature. The converse is true for a thin initial ice cover.

\section{GCM results}

The 2-D model results are relevant to the interpretation of global climate simulations. Pollard and Thompson (1994) summarize some of the aspects of including ice dynamics in their climate-change simulations, albeit with prescribed winds (the GENESIS version 1 runs discussed below). They find that the decrease in Arctic ice extent in a $2 \times \mathrm{CO}_{2}$ scenario is affected only slightly if a DT ice model is used. However, as predicted by Hibler and Ackley (1983) in their comparison of the role of dynamics in simulating Antarctic ice, a larger effect occurs in the Antarctic duc to the ability of the dynamic model to simulate a more realistic (less extensive) ice cover in the control run.

The 2-D model results discussed earlier suggest that the comparison of these GENESIS version 1 TO and DT runs 
for the Arctic should consider the spatial distribution of the ice pack in the $1 \times \mathrm{CO}_{2}$ (control) case. In the GENESIS version 1 TO control run, ice thicknesses are too thin over much of the Arctic. The GENESIS DT ice cover is more realistic in terms of thickness, but with thickest ice in the Siberian, central and east Greenland Sea sectors of the Arctic rather than along the Canadian coast (Pollard and Thompson, 1994; fig. 1).

Based on the 2-D model results, the relatively thin initial ice cover in the GENESIS TO run should accentuate the response to the $2 \times \mathrm{CO}_{2}$ scenario. Since, in the DT run, ice is too thick in the regions that show the greatest sensitivity in the stand-alone runs, the DT response to $\mathrm{CO}_{2}$ doubling is probably underestimated. In both cases, the location of the remaining ice in the $2 \times \mathrm{CO}_{2}$ scenario is confined to the central Arctic rather than the Canadian Arctic which, as noted above, could affect the ice-albedo feedback in the model by modifying the sensitivity of the ice pack to warming.

The GENESIS version 2 runs, using interactivelymodeled winds, yield a more accurate spatial distribution of ice cover in the control DT run than was the case for the version 1 simulation, even though prescribed winds were used in this earlier version 1 run. Changes in ice cover in the $2 \times \mathrm{CO}_{2}$ scenario are similar to the 2-D ice model results, with ice extent decreasing in the Siberian Arctic and Greenland and Barents Seas. Perhaps due to the more realistic ice conditions, the resulting net decrease in ice extent is greater in the version 2 DT results than in the v.l simulations. The GENESIS version 2 TO runs have not yet been completed, but preliminary results from the $2 \times \mathrm{CO}_{2}$ experiment suggest a greater response of the version 2 TO ice cover than was the case in the version 1 experiment. Initial ice thicknesses are probably similar to those in the version 1 ice cover, but with GENESIS version 2, ice is thickest near the Canadian Arctic rather than at the North Pole. This more realistic ice cover may be more sensitive to the $2 \times \mathrm{CO}_{2}$ climate perturbation.

\section{SUMMARY AND CONCLUSIONS}

Simulations using inter-annually varying atmospheric forcings allow us to address the four questions posed in the introduction. For the years studied, inter-annual variability in mean Arctic ice extent and ice volume is due primarily to ice transport, and the response of the ice cover to climate perturbations varies as a function of large-scale ice transport patterns. Interpretations of trends in observed ice cover in relation to global warming must therefore consider the role of atmospheric circulation and ocean currents in modifying the sensitivity of ice extent to changes in the energy budget. In addition to the role of inter-annual variations in ice advection, the overall response of the Arctic ice cover to climate perturbations is sensitive to the configuration of the pack in terms of ice-thickness patterns. Since ice dynamics contributes to the spatial distribution of thickness, transport can affect the nature of the response to climate change.

Based on the model and conditions used here, ice conditions simulated using the DT ice model are, overall, more sensitive than the TO results to small air temperature and flux perturbations, but less sensitive to large ones. This difference is a function of initial ice conditions including thickness distributions and spatial patterns, and decreases for a thinner initial TO ice cover.

Comparison of GCM runs indicates that differences in simulated ice response to doubled $\mathrm{CO}_{2}$ using different model versions is related, at least in part, to initial ice conditions and to the ability of the model to reproduce realistic spatial patterns of ice extent and thickness. GCM results in terms of ice-cover changes and sensitivity to the ice-albedo feedback appear quite sensitive to the spatial distribution of ice extent and thickness in the control runs. Simulations of the sensitivity of sea ice to global warming, and interpretations of previous GCM results, thus depend on a GCM's ability to reproduce relatively subtle features of the ice pack.

\section{ACKNOWLEDGEMENTS}

This work was supported by NSF grants OPP-932/547, OPP9423546 and OPP-9614297. Thanks are due to the NCEP/ NCAR project, and to D. Pollard at NCAR for GENESIS output and assistance with GENESIS version 2 runs.

\section{REFERENCES}

Crutcher, H. L. and J. M. Meserve. 1970. Selected level heights, temperatures and dew points for the Northern Hemisphere. Revised edition. Washington, DC, U.S. Navy. Weather Service Command. (NAVAIR Report 50-1C-52.)

Ebert, E. E. and J. A. Curry. 1993. An intermediate one-dimensional thermodynamic sea ice model for investigating ice atmosphere interactions. 7. Geophys. Res., 98 (C6), 10,085-10,109.

Flato, G. M. and W. D. Hibler, III. 1992. Modeling pack ice as a cavitating fluid. J. Phys. Oceanogr., 22 (6), $626-651$.

Flato, G. M. and W. D. Hibler, III. 1995. Ridging and strength in modeling the thickness distribution of Arctic sea ice. F. Geophys. Res., 100 (C9), $18,611-18,626$.

Hibler, W. D., III. 1979, A dynamic thermodynamic sea ice model. J. Phys. Oceanogr., 9 (7), 815-846.

Hibler, W. D., III and S. F. Ackley. 1983. Numerical simulation of the Weddell Sea pack ice. f. Geophys. Res., 88(C5), 2873-2887.

Hibler, W. D., III and K. Bryan. 1987. A diagnostic ice-ocean model. J. Phys. Oceanogr., 17 (7), 987-1015.

Holland, D. M., L. A. Mysak, D. K. Manak and J. M. Oberhuber. 1993. Sensitivity study of a dynamic thermodynamic sea ice model. 7. Geophys. Res., 98 (C2), 2561 - 2586.

Kalnay, E. and 21 others. 1996. The NCEP/NCR 40-year reanalysis project. Bull. Am. Meteorol. Soc., 77(3), 437-471.

Maslanik, J. A. and J. Key. 1995. On treatments of fetch and stability sensitivity in large-area estimates of sensible heat flux over sea ice. J. Geophys. Res., 100 (C3), 4573-4584.

Maslanik, J. A., D. McGinnis, M. Serrer, J. Dunn and E. Law-Evans. 1996a. An assessment of GENESIS V. $2.0 \mathrm{GCM}$ performance for the Arctic. In Arctic System Science Workshop, Boulder, CO, January 15-16 1995. Proceedings. Fairbanks, AK, Arctic Research Consortium of the United States, Tab-10, 7-8.

Maslanik, J. A., M. C. Serreze and R. G. Barry. 1996b. Recent decreases in Arctic summer ice cover and linkages to atmospheric circulation anomalies. Geophys. Res. Lett., 23 (13), 1677-1680.

Pollard, D. and S. L. Thompson. 1994. Sea-ice dynamics and $\mathrm{CO}_{2}$ sensitivity in a global climate model. Atmosphere-Ocean, 32 (2), 449-467.

Serreze, M. C., J. A. Maslanik, J. R. Key, R. F. Kokaly and D. A. Robinson. 1995. Diagnosis of the record minimum in Arctic sea ice during 1990 and associated snow cover extremes. Geophys. Res. Lett., 22 (16), 2183-2186.

Walsh, J. E. and H. J. Zwally. 1990. Multiyear sea ice in the Arctic: modeland satellite-derived. 7. Geophys. Res., 95(C7), 11,613-11,628.

Walsh, J. E., W. D. Hibler, III and B. Ross. 1985. Numerical simulation of Northern Hemisphere sea ice variability, 1951-1980. 7. Geophys. Res., 90 (C3), $4847-4865$. 